Cahiers de recherches médiévales

\title{
Christine de Pizan lectrice de Gilles de Rome
}

Le De Regimine principum et le Livre des fais et bonnes meurs du sage roy Charles $\mathrm{V}$

\section{Daisy Delogu}

\section{(2) OpenEdition}

\section{Journals}

Édition électronique

URL : https://journals.openedition.org/crm/10852

DOI : $10.4000 / \mathrm{crm} .10852$

ISSN : 1955-2424

Éditeur

Honoré Champion

Édition imprimée

Date de publication : 10 décembre 2008

Pagination : 213-224

ISSN : $1272-9752$

Référence électronique

Daisy Delogu, «Christine de Pizan lectrice de Gilles de Rome », Cahiers de recherches médiévales [En ligne], 16 | 2008, mis en ligne le 15 décembre 2011, consulté le 15 décembre 2022. URL : http:// journals.openedition.org/crm/10852 ; DOI : https://doi.org/10.4000/crm.10852 


\title{
rM
}

\section{Christine de Pizan lectrice de Gilles de Rome : Le De Regimine principum et le Livre des fais et bonnes meurs du sage roy Charles $V$}

\begin{abstract}
The compositional technique of compilation allowed Christine de Pizan to exploit the authority of her prestigious sources, and to transmit their teachings to new reading publics. The present article examines the use Christine made of Giles of Rome's De Regimine principum in her biography of Charles $V$ in order to demonstrate that compilation was more than "cut and paste», and to reveal the workings of an interpretive process that was dynamic and highly creative.

Résumé: La technique de la compilation a permis à Christine de Pizan de profiter de l'autorité de ses sources prestigieuses, et de transmettre leurs enseignements aux nouveaux publics de lecteurs. Ce travail examinera l'emploi qu'a fait Christine du De Regimine principum de Gilles de Rome dans sa biographie de Charles $V$ afin de montrer que la compilation était plus que «cut and paste » et pour dévoiler le fonctionnement d'un processus d'interprétation qui était dynamique et fortement créateur.
\end{abstract}

Depuis longtemps, les chercheurs ont reconnu que dans la rédaction de ses propres œuvres Christine de Pizan s'était servie de diverses sources classiques et médiévales. Certains critiques ont reproché à l'écrivaine prolifique un manque d'originalité, tandis que d'autres l'ont tout bonnement accusée de plagiat'. Or, l'usage que fait Christine de la citation, de la paraphrase, de la traduction, et de la réécriture des œuvres préexistantes la situe dans une tradition littéraire médiévale dont une des données de base est un respect profond pour le passé - qui renferme tout ce qui a de la valeur - et qui sert d'assise à sa propre auctoritas. Continuer, traduire (au sens tant linguistique que formel, ainsi que dans les maintes mises en prose, ou bien versifications, des textes), réécrire, étaient les activités primordiales de la création littéraire. Ces critiques de Christine, celles de ses contemporains comme celles des modernes, s'expliquent peut-être alors davantage par le fait qu'elle soit une femme-écrivain qui ose aborder les sujets de la politique, de l'éthique, et de la chevalerie, que par les techniques qu'elle emploie, techniques qu'elle partage, d'ailleurs, avec de nombreux autres écrivains de son époque.

Dans un passage du Livre des fais et bonnes meurs du sage roy Charles $V$ (dorénavant le Charles $V$ ) qui a fait couler beaucoup d'encre, passage qui préfigure

\footnotetext{
${ }^{1}$ Un des critiques les plus sympathiques à Christine a écrit: «Nous nous garderons donc de lui attribuer ou de lui demander des idées originales dans ce domaine, dans lequel son activité inlassable s'est bornée à répéter des notions plus ou moins connues et acceptées, à peine nuancées, ça et là, par sa sensibilité de femme». G. Mombello, "Quelques aspects de la pensée politique de Christine de Pisan d'après ses œuvres publiées », Culture et politique en France à l'époque de l'Humanisme et de la Renaissance, dir. F. Simone, Turin, Accademia delle Scienze, 1974, p. 43-153, esp. 152.
}

Cahiers de Recherches Médiévales, 16, 2008 
l'affirmation de Pascal que l'ordre contribue à la création du sens ${ }^{2}$, Christine explique et justifie la technique de la compilation et son emploi des sources antérieures. Elle travaille, nous dit-elle, comme l'architecte ou le brodeur, qui, eux, ne façonnent pas les matériaux avec lesquels ils œuvrent, mais qui les transforment en un objet qui est à la fois original et de grande valeur ${ }^{3}$. En réalité, je pense que l'usage que fait Christine de ses sources est même plus complexe qu'elle ne le dit. L'image des pierres ou des fils suggère une mosaïque textuelle dans laquelle l'auteur a rassemblé les morceaux qu'elle aurait trouvés ailleurs. Je trouve cependant que l'emploi qu'elle fait de ses sources évoque davantage la métaphore de Du Bellay de l'ingestion ${ }^{4}$, métaphore selon laquelle l'écrivain incorpore, dans le sens le plus littéral du terme, des œuvres antérieures, pour les intégrer, et pour les faire apparaître ensuite dans ses propres œuvres sous une forme entièrement nouvelle ${ }^{5}$.

Dans l'introduction à son édition du Charles $V$, Suzanne Solente parle des sources de Christine, tant orales qu'écrites, parmi lesquelles la Relation latine de la mort de Charles V, le Speculum historiale de Vincent de Beauvais, un Commentaire sur la Métaphysique d'Aristote, les Grandes Chroniques de France, une traduction des Flores chronicorum de Bernard Gui, et la traduction française effectuée par

\footnotetext{
${ }^{2}$ Dans la Pensée 22 Pascal écrit «Qu'on ne dise pas que je n'ai rien dit de nouveau : la disposition des matières est nouvelle; quand on joue à la paume, c'est une même balle dont joue l'un et l'autre, mais l'un la place mieux ». CEuvres de Blaise Pascal : publiées suivant l'ordre chronologique avec documents complémentaires, introductions et notes, éd. L. Brunschvicg et P. Boutroux, Paris, Hachette, 1904-1914, réimpr. Nendeln, Liechtenstein, Kraus Reprint, 1976-8.

3 «Tout ainsi comme l'ovrier de architecture ou maçonnage n'a mie fait les pierres et les estoffes, dont il bastist et ediffie le chastel ou maison, qu'il tent à perfaire et où il labeure, non obstant assemble les matieres ensemble, chascune où elle doit servir, selon la fin de l'entencion où il tent...tout ainsi vrayement n'ay je mie fait toutes les matieres, de quoy le traittié de ma compilacion est composé ; il me souffist seulement que les sache appliquer à propos, si que bien puissent servir à la fin de l'ymaginacion, à laquelle je tends à perfaire " (1, 191, c'est moi qui souligne). Toutes les citations viennent de Christine de Pisan. Le Livre des fais et bonnes meurs du sage roy Charles V, éd. S. Solente, Paris, Champion, 1936-1940, 2 vol., ci-après : $L F B M$ numéro de tome, numéro de page.

${ }^{4} \mathrm{Au} 3 \mathrm{e}$ chapitre, "Comment les Romains ont enrichi leur langue», de sa Défense et illustration de la langue française, Du Bellay écrit que «Imitant les meilleurs auteurs grecs, se transformant en eux, les dévorant, et après les avoir bien digérés, les convertissant en sang et nourriture, se proposant, chacun selon son naturel et l'argument qu'il voulait élire, le meilleur auteur, dont il observait diligemment toutes les plus rares et exquises vertus...». Joachim Du Bellay, Les Regrets précédé de Les Antiquités de Rome et suivi de La Défense et Illustration de la Langue française, éd. S. de Sacy, Paris, Gallimard, 1967, p. 214.

${ }^{5}$ Voir, sur cette question, la notion de 'compilation organique' introduite par J. Blanchard, "Compilation et légitimation au $\mathrm{XV}^{\mathrm{e}}$ siècle », Poétique, 74, 1988, p. 139-57, nuancée et 'revisitée' par B. Ribémont, "Christine de Pizan écrivain didactique: la question de l'encyclopédisme », Christine de Pizan, une femme de lettres, une femme de sciences, Actes du colloque de Liège janvier 2005, (dir. J. Dor et M.-E. Henneau), Paris, Champion, coll. Études christiniennes, 2008, p. 71-94.
} 
Henri de Gauchy du De Regimine principum de Gilles de Rome ${ }^{6}$. S. Solente analyse de façon attentive et précise l'emploi que fait Christine de ces sources, en juxtaposant des passages dans lesquels Christine transcrit ou paraphrase sa source. C'est cette méthode de compilation qui ressemble le plus à la mosaïque, ou bien à la broderie ou l'architecture. Les segments isolés d'un texte peuvent être délimités et leurs origines retracées. Le processus d'écriture de Christine se compose cependant d'une variété de techniques, dont plusieurs sont bien plus complexes que celle-ci. Dans ce travail nous considérerons les particularités de l'usage que fait Christine de la compilation dans sa biographie de Charles $\mathrm{V}$, étude qui nous permettra de mieux comprendre les processus de lecture et d'écriture médiévales. À cette fin nous nous concentrerons sur une de ses sources les plus importantes, point de repère essentiel, le De Regimine principum.

Le De Regimine principum de Gilles de Rome, commandé par Philippe III entre 1277 et 1281 et dédié à son fils, le futur Philippe le Bel, était un des Miroirs $d u$ prince les plus importants du Moyen Âge, ainsi que le démontre le nombre important de manuscrits qui nous sont parvenus, plus que $350^{7}$; Gilles faisait partie des écrivains qui ont tenté, suite à la traduction gréco-latine de Guillaume de Moerbeke de la Politique d'Aristote, d'expliquer et de diffuser les écrits du Philosophe, et de réconcilier sa pensée avec l'enseignement de l'Église 8 . L'œuvre de Gilles se divise en trois livres - le gouvernement de soi, de la maison, et du royaume -, dont chacun relève d'une de ses sources aristotéliciennes: l'Éthique, l'Économique et la Politique, respectivement.

Le De Regimine principum fut traduit presque immédiatement en français par Henri de Gauchy, et des générations de rois, tant Valois que Capétiens, ont continué à posséder et à commander les deux versions du texte. Pendant le règne de Charles $\mathrm{V}$, la bibliothèque royale renfermait une douzaine d'exemplaires du De Regimine principum en latin et en français, que Christine aurait pu consulter lors de

\footnotetext{
${ }^{6}$ Pour la plupart, S. Solente suit les travaux d'Henri Duchemin, dont la thèse de 1891 à l'École des Chartes se concentre sur les sources de Christine dans le Charles V. Voir LFBM 1, xxxii-lxxx.

${ }^{7}$ Sur le Miroir du prince voir J. Blanchard et J.-C. Mühlethaler, Écriture et pouvoir à l'aube des temps modernes, Paris, Presses Universitaires de France, 2002, p. 7-32; K. Langdon Forhan, "Reflecting Heroes: Christine de Pizan and the Mirror Tradition », The City of Scholars : New Approaches to Christine de Pizan, dir. M. Zimmermann et D. De Rentiis, New York et Berlin, de Gruyter, 1994, p. 189-96; J. Krynen, L'Empire du roi, idées et croyances politiques en France : XIII ${ }^{e}-X V^{e}$ siècle, Paris, Gallimard, 1993 ; et C. Nederman, "The Mirror Crack'd: The Speculum principum as Political and Social Criticism in the Late Middle Ages ", The European Legacy, 3.3, 1998, p. 18-38. Sur le De Regimine principum voir C. Briggs, Giles of Rome's "De Regimine principum" : Reading and Writing Politics at Court and University, Cambridge, Cambridge University Press, 1999.

${ }^{8}$ Sur la réception d'Aristote, surtout la Politique, au Moyen Âge tardif, voir J. Blythe, Ideal Government and the Mixed Constitution in the Middle Ages, Princeton, Princeton University Press, 1992; J. Dunbabin, "The Reception and Interpretation of Aristotle's Politics», Cambridge History of Later Medieval Philosophy, dir. N. Kretzmann, A. Kenny et J. Pinborg, Cambridge, Cambridge University Press, 1982, p. 723-37 et C. Richter Sherman, Imaging Aristotle: Verbal and Visual Representation in Fourteenth-Century France, Berkeley, University of California Press, 1995.
} 
sa rédaction de la biographie du roi sage ${ }^{9}$. Puisqu'elle cite exclusivement dans le Charles $V$ la version française de Gilles de Rome, le Livre du regisme des princes, c'est à cette version que nous ferons référence au cours de cet article ${ }^{10}$.

La vie de Charles V qu'a écrite Christine ne se conforme pas exactement à nos attentes pour un texte biographique ${ }^{11}$. D'abord, le Charles $V$ ne procède pas de façon chronologique, comme nous avons tendance à concevoir un projet biographique. Ce texte est, en revanche, constitué de trois livres - tout comme le Regisme des princes - dont chacun se focalise sur une qualité précise : noblesse de courage, chevalerie, et sagesse. Christine parle de manière théorique de chacune de ces qualités et des parties qui les constituent, et elle démontre également comment Charles illustrait la qualité donnée. Ainsi le Charles $V$ est fondamentalement dialogique dans sa méthode, engagé dans un va et vient constant entre l'abstrait et le particulier, l'hypothétique et l'historique. De cette manière, Christine combine les desseins biographiques et didactiques, en retraçant la vie de Charles $\mathrm{V}$ et en fournissant en même temps un modèle du roi idéal. Ce dialogue s'instaure au niveau du texte même de Christine, ainsi que, comme nous le verrons, entre sa biographie et sa source.

La manière la plus notable dont Christine se sert du texte de Gilles est de procéder par citation. Cette technique se manifeste surtout au cours du livre II du Charles $V$, consacré à la chevalerie. Au troisième livre du De Regimine principum, qui traite de la gouvernance du royaume en temps de paix comme en temps de guerre, Gilles, qui, lui, suit de près l'historien et théoricien militaire Vegèce, fournit une sorte de manuel pour les chefs militaires et politiques dans lequel il donne des conseils concrets et pratiques sur une grande variété de sujets : quelle sorte de gens font les meilleurs chevaliers, l'âge auquel on doit commencer l'entraînement chevaleresque, comment fabriquer les machines de guerre, comment choisir les champs de bataille les plus avantageux, et ainsi de suite.

Christine fournit les mêmes informations dans le deuxième livre de son ouvrage, en s'appuyant souvent et de façon explicite sur le texte de Gilles; mais le contexte au sein duquel elle présente cette matière n'est pas du tout le même. Gilles s'adonne à une analyse systématique de la guerre au niveau théorique, tandis que les enseignements de Christine participent d'un dialogue entre la théorie et la pratique. Ce va-et-vient entre l'abstrait et le concret se remarque à tous les niveaux de son texte, des titres qu'elle donne aux chapitres, qui sont tous contextualisés historiquement, à la forme même des chapitres, qui juxtaposent un principe de l'art militaire, souvent tiré du texte de Gilles, à son application par Charles V. Dans le chapitre qui s'intitule «Comment le roy Charles fist messire Bertran de Clequin son connestable » (II, 19), Christine, en citant l'œuvre de Gilles, explique à ses lecteurs

\footnotetext{
${ }^{9}$ L. Delisle, Recherches sur la librairie de Charles V, Paris, 1907, Amsterdam, G. Th. van Heusden, 1967, t. 2, p. 87-88.

${ }^{10} \mathrm{~S}$. Solente se range à l'avis d'H. Duchemin, qui affirme que Christine s'est servie de la version française et non pas de la version latine du texte de Gilles, bien que les deux se soient trouvées dans la bibliothèque du Louvre ( $L F B M 1$, lxiv).

${ }^{11}$ Nous avons proposé ailleurs que l'organisation thématique de Christine suit le modèle biographique suétonien, mais d'autres ont cité son manque de chronologie précise pour classer son texte comme étant autre qu'une biographie. Theorizing the Ideal Sovereign. The Rise of the French Royal Vernacular Biography, Toronto, University of Toronto Press, 2008.
} 
que « par moult grant esgart et deliberacion de sens doit estre avisié quelz gens on establist chevetains et conduiseurs des aultres » (LFBM 1, 185). Ensuite, elle justifie et loue le choix qu'avait fait Charles V en faisant de Bertrand du Guesclin son connétable malgré la modeste naissance de celui-ci, bien inférieure à celle des connétables précédents. Cette même structure se répète tout au cours du deuxième livre, dans lequel des épisodes de la reconquête des terres qui avaient été cédées à l'Angleterre à la suite du traité de Brétigny introduisent, illustrent des passages qui proviennent du troisième livre de Gilles sur l'art militaire et alternent avec eux. Ainsi, les discours théoriques et historiques se nourrissent mutuellement, car Christine profite de l'autorité de son modèle littéraire pour montrer la perspicacité de Charles $\mathrm{V}$ en matière de guerre, tandis que l'énumération des nombreux succès français démontre la sagesse des propos de Gilles. L'intercalation des conseils militaires de Gilles avec des exemples historiques qui proviennent du règne de Charles V montre comment la théorie influe sur la conduite politique et militaire, et suggère qu'elle doit continuer à le faire au moment de la composition de Christine. La théorie n'est pas isolée ou stérile, mais porte directement sur les événements historiques et politiques. Le dialogisme du texte de Christine donne un exemple à ses lecteurs de l'application de la théorie à la pratique qu'ils doivent incorporer dans leurs propres vies.

Christine emprunte au texte de Gilles bien plus que les informations techniques. Sa reformulation de l'idée même de chevalerie en tant qu'entreprise intellectuelle au lieu d'activité physique doit beaucoup à la pensée de son prédécesseur, qui écrit que «chevalerie est un espece et une maniere de sens et d'avisement par quoi l'en seurmonte les ennemis et ceus qui deffendent et empeschent le bien et le profit commun » $\left(\right.$ p. 372) ${ }^{12}$. Christine affirme à son tour que " chevalerie est une espece de sens et avis de surmonter les anemis et empeschieurs du bien commun » (LFBM 1, 115). Certes l'importance de sens et d'avisement ne fait pas négliger, pour Gilles, la prouesse physique, tandis que pour Christine, qui cherche à démontrer la chevalerie d'un roi physiquement fragile qui n'a jamais, après son couronnement, chevauché à la tête de ses armées, ces valeurs prédominent sans hésitation sur la simple force. Ainsi nous pouvons constater que tout comme la théorie oriente la pratique, de même les événements historiques influent sur les idées.

Dans d'autres parties du Charles $V$, Christine se réfère au texte de Gilles, mais en transformant subtilement son modèle afin de le mettre en accord avec l'application de ses principes par Charles V. En parlant de l'établissement des nouvelles lois par exemple, Christine cite avec approbation les modifications que Charles avait apportées aux lois de succession, modifications qui avaient pour objectif d'assurer la succession héréditaire continue et incontestée de la couronne (2, 22). Ce passage est suivi par une référence au Regisme des princes, dans lequel Gilles cite lui-même la Politique d'Aristote, là où le Philosophe donne quatre raisons qui montrent les avantages des changements de lois. Cependant Gilles, tout comme Christine, présente Aristote comme un homme de paille, car Gilles conclut

${ }^{12}$ Toutes citations, par numéro de page, viennent de Li Livres du gouvernement des rois. $A$ $X I I I^{\text {th }}$ century French version of Egidio Colonna's Treatise De Regimine principum now first published from the Kerr Ms, éd. S. P. Molenaer, New-York, Macmillan, 1899, réimpr. NewYork, AMS Press, 1966. 
que d'accepter les prémisses d'Aristote "seroit mult grant peril, quer se l'en acoustumoit a establir noveles loys, l'en acoustumera a cen que l'en n'obeira pas as loys » (p. 359). Il parle ensuite du lien entre l'habitude et l'obéissance, et il précise les circonstances dans lesquelles il est légitime pour le roi de modifier les lois (i. e., si elles contredisent la loi ou la raison naturelle [p. 360]), pour conclure qu'en principe, les lois ne doivent pas être changées ${ }^{13}$.

Dans son traitement de la loi, comme dans les passages sur l'art militaire, le même contenu apparaît dans deux contextes différents. Gilles fait une analyse de la loi en tant que telle, tandis que Christine parle de la loi par rapport à la «prudence et art en la personne du roy Charles » (LFBM 2, 21), et ses citations du texte de Gilles visent à éclairer et à louer les actes du sage roi. Christine se conforme à son modèle dans sa condamnation de ce passage d'Aristote et en reconnaissant que la loi positive ne peut pas contredire la loi naturelle. Pourtant, à la fin de ce chapitre (III, 6) et de sa discussion de la loi, Christine ajoute une dernière réflexion qui ne se trouve pas chez Gilles, à savoir l'affirmation que bien que les rois ne doivent pas changer la loi, tout de même " par bon esgart et au profit du bien commun adjouster y peut on » $(L F B M 2,25)$. Comme Christine mélange librement des passages tirés de ses sources avec ses propres pensées, il est difficile de savoir exactement où s'achève l'un et où commence l'autre. Cette ambiguïté voulue permet à Christine d'utiliser les enseignements de Gilles pour autoriser ce qui constitue, au moins potentiellement, une mise en retrait des conseils de celui-ci.

Toujours dans le cadre de son chapitre sur la prudence du roi Charles, Christine se sert d'un passage qui provient d'une autre partie du texte de Gilles pour justifier les modifications apportées par le monarque aux lois de succession. Christine commence avec une définition de la prudence, qui «par memoire des choses passées porvoie aux futures» $(2,21)$. Cette phrase rappelle très clairement le début du traité de Gilles, là où il explique que le roi doit conduire son peuple à de bonnes fins. Pour savoir quelles sont ces fins, Gilles explique à ses lecteurs qu'«il covient que li roi aient memoire des choses passees et porveance des choses a venir » (40). Christine présente les modifications de Charles sur les lois comme un exemple de memoire et de porveance - deux qualités qui sont essentielles pour les rois - dirigées au «bien de la couronne de France et de la commune utilité » $(2,22)$. Donc, en citant le Regisme des princes, Christine revendique pour elle-même l'autorité de son modèle, tandis qu'en réordonnant le texte de Gilles et en y faisant des ajouts, elle suggère que les actions de Charles furent en conformité parfaite avec l'idéal du roi que Gilles avait formé.

Bien que dans le Charles $V$ Christine cite le Regisme des princes quasiment plus que n'importe quelle autre œuvre, l'influence du texte de Gilles apparaitt également de façon plus indirecte au cours de sa biographie, dans des passages qui ne peuvent pas être isolés et comparés systématiquement. L'influence de Gilles se fait parfois sentir au fait qu'elle reconnaît avec lui l'importance d'une valeur particulière. Par exemple, un aspect essentiel de la philosophie de la vertu de Gilles,

\footnotetext{
${ }^{13}$ En fait Aristote nous fait remarquer l'utilité de l'habitude en ce qui concerne l'obéissance aux lois, et il dit que les lois doivent être modifiées seulement avec prudence. Voir Aristote, The Politics and the Constitution of Athens, éd. S. Everson, Cambridge, Cambridge University Press, 1996, p. 49.
} 
qu'il emprunte à Aristote, est l'idée de la mesure ou du juste milieu ${ }^{14}$. Les vertus et les vices ne se situent pas aux antipodes d'une échelle de la moralité. Au contraire, la vertu se définit chez Gilles comme un équilibre entre deux vices également blâmables. Ainsi le courage se trouve entre les extrêmes de la peur et de l'outrecuidance. Gilles définit de même la bonté et l'amabilité comme les manifestations convenables de la colère et de l'amour, respectivement, dans les paroles et dans les actes. Dans le Charles $V$, l'importance de l'ordre et du juste milieu se fait jour à travers l'idéal de l'ordre. On pourrait affirmer que la «belle ordenance » $(1,39)$ de Charles aurait pu définir celui-ci, sauf que Charles le bien ordonné n'a pas tout à fait la même résonance que Charles le sage. Le sentiment d'ordre de Charles permet de donner à chacun ce qu'il lui est dû, et constitue la base des vertus, comme la générosité, qui ne veut pas dire donner abondamment, mais donner de façon convenable à ceux qui le méritent, ou comme la tempérance, qui n'exige pas l'abstinence, mais l'indulgence au bon moment et au bon endroit. Grâce à son ordre, Charles se situe, et il situe tous ceux qui l'entourent, à leur place politique, morale, et physique, et cet idéal transparaît dans le Charles $V$ à travers le lexique de Christine, qui emploie avec une fréquence notable les mots tels que ordre, ordennance, et ordennés ${ }^{15}$.

L'ordre de Charles sert également à montrer sa capacité à régler sa vie. Puisque Gilles écrit que celui qui se veut roi doit d'abord savoir se gouverner, Christine décrit une journée typique du sage roi. Le fait même qu'il ait une journée typique nous fournit une première preuve du sens de l'ordre du roi, tandis que les éléments de cette journée témoignent chacun de son bon caractère et sa bonne conduite de soi, de sa maison, et de son royaume. Charles se lève avec le jour ${ }^{16}$, et au cours de la journée il consacre une partie de son temps et de son attention à Dieu, à ses sujets et à l'administration du royaume, à ses nobles, aux étrangers ou aux ambassadeurs, à sa femme et à ses enfants, et à son propre plaisir. Dans chaque circonstance, tant avec ses intimes qu'avec des étrangers, Charles « donnoit response par si moriginée maniere, et si deuement rendoit à chascun l'onneur, qu'il apartient, que tous s'en tenoient pour tres contens » $(L F B M 1,45)$. Christine insiste sur la modération et la justice des paroles, des gestes, et des actes de Charles vis-à-vis de tous ceux qu'il rencontre.

En examinant de près la manière dont Christine reprend l'œuvre de Gilles, on arrive à comprendre son interprétation de ce texte important. Elle amplifie énormément certains propos de son prédécesseur, tandis que d'autres sont

\footnotetext{
${ }^{14}$ Gilles nous dit que l'homme se situe entre les anges et les betes (p. 10).

${ }^{15}$ Voir en particulier la description que fait Christine des chevauchées de Charles, ou de la visite du saint empereur romain Charles IV. Sur l'idéal de l'ordre dans le Charles $V$ voir F. Autrand, «Mémoire et cérémonial: La visite de l'empereur Charles IV à Paris en 1378 d'après les Grandes Chroniques de France et Christine de Pizan », Une Femme de lettres au Moyen Âge: études autour de Christine de Pizan, dir. L. Dulac et B. Ribémont, Orléans, Paradigme, 1995, p. 91-103 ; et D. Delogu, « Reinventing the Ideal Sovereign in Christine de Pizan's Livre des fais et bonnes meurs su sage roy Charles V», Medievalia et Humanistica, 31, 2005, p. 41-58; et l'article précédent dans ce volume (S. Dudash).

${ }^{16}$ Le «bestornement» de la nature qui consiste à faire de la nuit le jour, et du jour la nuit, est l'objet des critiques de Charles VI et de sa cour que font, entre autres, Jean Gerson et Eustache Deschamps.
} 
considérablement réduits. L'exposé de Gilles sur les vertus personnelles que doit posséder le roi idéal se concentre dans la deuxième partie du premier livre (qui traite de la gouvernance de soi). La qualité la plus importante, condition sine qua non du gouvernement efficace, est la sagesse. Parmi les autres qualités du bon roi, on trouve la tempérance, le courage et la justice, suivis de la générosité, l'amour de l'honneur, et la courtoisie, ainsi que la magnificence, la magnanimité, la bonté, et l'amabilité. Gilles ordonne soigneusement les vertus, il définit les termes dont il se sert, et il explique les parties constituantes de chaque qualité. Il s'intéresse aux vertus même, à leur rapport les unes aux autres, et à la manière dont le roi sage peut les mettre en pratique. En revanche les qualités de noblesse de cœur, de chevalerie, et de sagesse fournissent à Christine la structure de son texte, tandis qu'elle parle ici et là au cours de sa biographie d'une foule d'autres vertus, dont le choix est manifestement inspiré par le traité de Gilles, toujours en rapport avec le règne de Charles V. Il est vrai que Christine parle de certaines vertus d'une manière abstraite, comme lorsqu'elle définit noblesse de cœur ou les cinq composantes de la sagesse, mais la plupart du temps c'est Charles qui lui fournit son point de départ, et ses actes deviennent un prétexte pour parler d'une vertu particulière. Bien que Christine ne traite pas des vertus dans le même ordre que Gilles, ou dans la même partie de son texte, il est néanmoins évident que la conception du roi idéal exposée par l'auteur du De Regimine principum transparaît dans la biographie de Christine, car elle réussit à démontrer comment Charles illustre toutes les qualités jugées nécessaires par Gilles, et même davantage. Le Charles $V$ fournit des exemples des multiples vertus du roi , tempérance, justice, clémence, bonté, humilité, générosité, chasteté, honnêteté, charité, dévotion, prudence, et bien sûr sagesse. En répondant ainsi au texte de Gilles, et en s'assurant d'avoir bien montré comment Charles avait manifesté chacune des qualités requises par le Regisme des princes, Christine fait de Gilles un interlocuteur privilégié. Le dialogue que nous avons remarqué entre la théorie et la pratique à l'égard de l'art militaire se produit ici entre les textes de Christine et de Gilles. Un lecteur qui connaissait le Regisme des princes, qui, ne l'oublions pas, aurait été commandé et lu par le même public que le Charles $V$, aurait pu y reconnaître les principes qui trouvent leur réalisation dans la conduite de Charles V, telle que la présente Christine.

$\mathrm{Si}$, dans ces passages Christine amplifie et diffuse les enseignements de Gilles en ce qui concerne les vertus royales, ailleurs elle réduit de façon remarquable ses recommandations. Au début du Charles $V$, dans une allusion transparente au triple intérêt qui structure l'œuvre de Gilles, elle dit que le roi idéal doit gouverner «soy-meismes, sa maisgné et subgiez et toutes choses selon ordre juste et limité » $(L F B M 1,52)$. Nous avons déjà constaté que Christine prenait soin de montrer comment Charles se comportait selon la raison et l'ordre. En outre, elle se penche sur sa gouvernance de la reine, Jeanne de Bourbon, affirmant que dans ses vêtements et ses parures, ses plaisirs et ses compagnons, son manger et son boire, la reine a toujours maintenu un équilibre parfait entre la sobriété et la magnificence $(1$, 53-57). Christine est plus circonspecte pour ce qui touche aux enfants royaux, qui, au moment de la rédaction de son ouvrage, ne menaient pas une vie à la hauteur de l'exemple paternel. Elle dédie néanmoins un chapitre à «la nourriture et discipline de ses enfans » $(1,57)$ dans lequel elle déclare que Charles avait placé ses enfants sous la conduite de bons chevaliers et de maitres sages. Quant à son royaume, 
Charles « ses princes et nobles maintenoit en honneur et largece et de lui contens; le clergié tenoit en paix, le peuple en crainte et obeissance en temps de paix et de guerre, les estranges nacions benivolens » $(1,52)$. Ainsi dans les premiers chapitres de sa biographie, Christine montre de manière indiscutable la gouvernance exemplaire du roi de lui-même, de sa maison, et de son royaume. Avec cette référence au traité de Gilles, qui est à la fois une reprise et une diminution de son modèle, Christine reconnaît et congédie simultanément son prédécesseur, pour faire place à son propre portrait de Charles $\mathrm{V}$ et du roi idéal.

Le point de contact peut-être le plus intéressant entre Christine et Gilles n'a rien à voir avec la transmission des informations techniques, ni avec les principes conceptuels et structurants qui gouvernent leurs œuvres, ni même avec l'idée du roi modèle, mais relève de la manière dont chaque auteur souligne le poids des résultats, et la nécessité de mettre en œuvre les connaissances que l'on possède. Il n'est pas question de la citation, ni de l'inspiration indirecte. Il s'agit plutôt d'une préoccupation globale des deux auteurs, et on n'a pas moyen de savoir si Christine a suivi l'augustin à cet égard, ou si chez Gilles elle a reconnu tout simplement un esprit en sympathie avec le sien. Dans l'introduction à son texte, Gilles demande, de façon rhétorique, de quelle manière on peut mieux transmettre les enseignements moraux et politiques aux rois et aux princes, et il conclut que « la maniere du parlier en ceste science est grosse et par essanple» (p. 4). Il y a plusieurs raisons. D'abord les raisonnements subtils illuminent l'intellect ou la compréhension (l'«entendement»), tandis que les exemples remuent la volonté (l'«entalentement»), en provoquant le désir de suivre l'exemple donné (p. 5). En outre, bien que le Regisme des princes s'adresse principalement à un public noble, il est également utile pour les gens humbles, mais comme le peuple n'est pas capable de suivre un raisonnement compliqué, il leur faut des exemples. En effet, le roi luimême doit servir d'exemple de la vertu à son peuple (p. 85), si bien qu'il devient une sorte de texte vivant qui incarne et projette les principes développés par Gilles. Charles V est un tel roi, et la biographie de Christine effectue précisément l'inverse du processus proposé par Gilles, en transformant les faits exemplaires et le caractère du roi sage en un discours qu'elle présente explicitement comme un exemple pour ses lecteurs. La vie de Charles V sert donc de relais dans la double articulation des actes et du texte, qui s'influencent mutuellement.

L'insistance de Gilles sur l'importance des exemples et l'exemplarité va de pair avec sa hiérarchisation de la pratique sur la théorie. On peut remarquer à cet égard son admiration pour l'Éthique d'Aristote, où le Philosophe distingue les connaissances scientifiques (episteme) de la sagesse pratique (phronesis). Le privilège accordé à cette dernière pourrait remonter à Cicéron, pour qui la plus haute sagesse est celle qui a pour objectif un résultat concret ${ }^{17}$ On peut mesurer la distance qui sépare le savoir et le faire dans les passages qui parlent d'une connaissance précise. En ce qui concerne la loi par exemple, Gilles dit que «il ne soufist pas a savoir les loys et les costumes, se on ne les siet apliquier as euvres humaines » (p. 42). De même en parlant de l'administration de la justice, il écrit que l'expérience

${ }^{17}$ Voir R. Copeland, "the highest wisdom is that which has its end in result», Rhetoric, Hermeneutics, and Translation in the Middle Ages, Cambridge, Cambridge University Press, 1991, p. 17. 
est plus utile que la formation scolaire, car ceux qui possèdent la première «puent mieuz jugier que ceus qui ont la science des lois » (p. 341).

Gilles fait preuve d'un pragmatisme semblable quand il parle de la morale en général. Cela est parce que «nos n'aprenon pas la science de bones meurs principaument por savoir, ainz l'aprenon principaument por ce que nos soion boens » (p. 5, c'est moi qui souligne). Cette attitude explique pourquoi, tout au long de son texte, Gilles parle non pas de la sagesse, mais des oeuvres de sagesse. La première partie du premier livre de Gilles est consacrée au souverain bien qui consiste à faire «euvres de vertu» (p. 24) et à connaître Dieu. On ne peut pas dissocier la sagesse de la volonté et des actes. Un individu peut être «soutif et malicieus et de bon engyn, mes il ne puet estre que li hons soit sage [...] se il n'est de bone vie et face les euvres de vertu » (p. 37). La sagesse n'est donc pas une qualité que l'on possède, mais que l'on réalise. Les connaissances toutes seules ne suffisent pas, et la sagesse, pour Gilles, n'est pas l'équivalent du savoir; au contraire, la sagesse s'oriente toujours vers les objectifs concrets. À chaque pas, il souligne la nature performative des qualités qu'il cherche à inculquer à son public royal. Il ne cherche pas à obtenir de ses lecteurs une reconnaissance abstraite des qualités qui mènent à la bonne gouvernance du royaume, mais une réalisation consciente et active de la conduite digne d'un roi $^{18}$. L'insistance de Gilles sur les actions suppose un lecteur engagé, pour qui son texte ne serait pas une fin mais un point de départ. Si Gilles nous livre un modèle du roi idéal, et de la moralité au sens large, les bonnes œuvres auxquelles il fait sans cesse référence ne se réalisent pas au sein de l'espace discursif de son texte, mais attendent leur concrétisation dans la vie de ses lecteurs. Le texte de Gilles fonctionne ainsi comme un interlocuteur qui fournirait une moitié d'une conversation morale et politique dont l'autre moitié serait du côté du lecteur, et aussi comme un exemplum qui, comme dans les textes hagiographiques, inviterait à l'imitation du texte ${ }^{19}$.

L'accent que Gilles met sur les actes et les conséquences est une présupposition fondamentale pour le Charles $V$, tant par rapport à la reformulation de l'idée du roi idéal effectuée par Christine que pour son propre statut d'auteur. L'intérêt de Christine pour les conséquences se voit tout au long de sa biographie, mais nulle part de façon aussi remarquable que dans le Livre 2 , où elle montre que ce roi fragile qui, après son couronnement n'a jamais chevauché à la tête de ses armées, était en réalité un «vray chevalereux» $(L F B M 1,243)$. Christine peut formuler cette affirmation paradoxale grâce à une redéfinition de la chevalerie même, qui ne consiste plus à mener son armée, mais à posséder les qualités de «bonne fortune, sens, diligence, et force» $(1,117)$. Que Charles soit en possession de ces vertus « nous tesmoigne et fait certains la vraye experience, que nous sçavons clerement par la fin de ses glorieuses conquestes » $(1,118)$. Christine présente cette

\footnotetext{
${ }^{18}$ M. Richarz dit que « [t] his realization of wisdom in action is called prudence ». « Prudence and Wisdom in Christine de Pizan's Le Livre des fais et bonnes meurs du sage roy Charles $V$ », Healing the Body Politic. The Political Thought of Christine de Pizan, éd. K. Green et C. Mews, Turnhout, Brepols, 2005, p. 99-116, p. 102.

${ }_{19}$ Sur les pratiques médiévales de lecture voir J. Dagenais, The Ethics of Reading in Manuscript Culture : Glossing the Libro de buen amor, Princeton, Princeton University Press, 1994, et B. Stock, Listening for the Text: On the Uses of the Past, Philadelphia, University of Pennsylvania Press, 1990.
} 
conclusion comme allant de soi. De même, elle nous dit que la «perfaitte prudence » $(2,21)$ du sage roi « appert par les effetz de ses oeuvres » $(2,21)$. Au lieu donc de chercher l'expression de ses vertus dans la personne de Charles, Christine se tourne du côté de leurs manifestations extérieures, ses faits et ses succès, qui lui permettent, dans un processus d'induction logique, d'affirmer qu'il possède incontestablement les qualités du roi idéal.

La même tendance à privilégier les effets plus que la source et les actions plus que l'acteur donne lieu, chez Christine, à la revendication de sa propre autorité de femme-écrivain. En tant que femme qui ose écrire sur la fonction royale et sur la chevalerie, Christine sait bien que son autorité est vulnérable. Tout comme elle l'avait fait pour prouver la chevalerie et la sagesse de Charles, elle souligne la qualité de ses propres travaux pour revendiquer son autorité d'écrivain. On ne devrait pas juger la personne qui écrit, dit-elle, mais la valeur de ce qui est $\operatorname{dit}^{20}$. Pour Christine comme pour Gilles, les effets, les résultats, et les actions fournissent l'aune à laquelle on peut juger un individu.

Cette mise en valeur des actes et des conséquences peut mener à un pragmatisme quelque peu surprenant. Dans sa taxonomie de la vertu par exemple, Gilles explique « quele chose est verité » (p. 87). Selon Gilles, la vérité demande de la cohérence, en paroles et en faits, entre le for intérieur d'un individu, et son apparence extérieure. Une personne honnête ne se présente pas autrement que ce qu'elle est. Une telle honnêteté exige une évaluation franche et une représentation fidèle de soi-même. Gilles reconnaît cependant que « ja soit cen que nus ne doie mentir, toute voies chascune verité n'est pas bone a dire touz jorz par tout, mes l'en puet taire verité en tens et en liu selon l'engovernement de reson» (p. 88). Donc cacher la vérité ne constitue pas la malhonnêteté, et une telle dissimulation est à louer dans certaines circonstances, à déterminer avec l'usage de la raison. L'énoncé de Gilles nous révèle son attitude particulière envers la vérité, le langage, et la production du sens selon laquelle la vérité n'est pas un bien ontologique, ni une entité fixée ou immanente dans le langage. La valeur de la vérité dépend des circonstances dans lesquelles elle est prononcée ${ }^{21}$. Dans le Charles $V$, Christine évoque le point de vue très semblable de Charles sur la question de la dissimulation. À certaines personnes de son entourage, qui défendaient l'opinion selon laquelle la dissimulation constitue une sorte de trahison, Charles a expliqué que «les circonstances font les choses bonnes ou mauvaises, car en tel maniere peut estre dissimulé, que c'est vertu, et en tel maniere, vice » $(2,74)$. Comme dans les passages où Christine et Gilles demandent l'engagement de leurs lecteurs, nous observons de nouveau qu'ici, dans leur réflexions sur la vérité et la dissimulation, les deux auteurs rejettent l'idée d'une finitude ou d'une fixité du sens qui réside dans le texte même (que ce soit un texte écrit ou oral). Le tout serait de prendre en compte les

\footnotetext{
${ }^{20}$ Christine s'était servie du même procédé lors de la querelle du Roman de la rose. Tandis que ses adversaires dans ce débat littéraire ont invoqué systématiquement l'autorité personnelle de Jean de Meun pour prouver la qualité de son texte, Christine en revanche a tenté de réorienter ce débat pour le faire focaliser sur l'œuvre même. Voir Le Débat sur le Roman de la Rose, éd. É. Hicks, Paris, Champion, 1977.

${ }^{21}$ On peut mettre en parallèle cette perspective avec celle des rhétoriciens médiévaux qui ont mis en relief l'importance du contexte dans l'établissement d'une interprétation. Sur la grammaire et la rhétorique médiévales, voir R. Copeland, op. cit. et B. Stock, op. cit.
} 
circonstances, y compris les lecteurs ou les auditeurs, pour contribuer à la création du sens.

Chez Gilles de Rome, Christine a trouvé non seulement une source d'information technique ou un modèle du roi idéal, mais un interlocuteur précieux. La facilité avec laquelle Christine cite, paraphrase, réordonne, fait écho et répond au De Regimine principum nous montre jusqu'à quel point l'œuvre de Gilles fût incorporée dans sa propre pensée. L'usage qu'elle fait du texte de Gilles n'est pas un acte d'imitation servile, mais de transformation et de régénération. De l'ancien elle fait du nouveau, de l'étrange elle fait l'intime. C'est un testament de l'intérêt durable de l'œuvre de Gilles auprès des publics médiévaux, et également de la culture littéraire profonde de Christine. D'une part on peut envisager le Charles $V$ comme une sorte de réponse au Regisme des princes et à la focalisation de Gilles sur les faits, les résultats, et la pratique; d'autre part il ne s'agit pas d'un dialogisme diachronique, où Christine ajouterait simplement et tardivement au texte de Gilles. $\mathrm{Au}$ contraire, elle institue un dialogue entre les deux textes, et elle actualise au niveau de son texte une lecture engagée et morale (il s'agit bien de l' «ethical reading » de Dagenais) telle qu'elle et Gilles aimeraient la voir se réaliser chez leurs lecteurs. Le dialogisme que nous avons observé au sein du texte de Christinecomme dans les passages sur l'art militaire, scandés par les exemples du règne de Charles V - se reproduit entre les textes de Gilles et de Christine. Il se produit également entre le texte et la vie, comme lorsque Charles traduit les recommandations du Regisme des princes et d'autres textes moraux et politiques en pratique royale ${ }^{22}$. Et quand Christine à son tour transforme les particularités de la vie du sage roi en un miroir du prince. Nous voyons que la technique de la compilation telle qu'elle est employée dans le Charles $V$, ainsi que dans la culture littéraire dont Christine a fait partie, est, comme la lecture et la traduction - voire comme la vie elle-même - un processus dynamique d'interprétation et de création.

\section{Daisy Delogu \\ University of Chicago/USA}

\footnotetext{
${ }^{22}$ J'emploie le terme «traduit» sciemment, car l'analyse de R. Copeland au sujet de la pratique médiévale de la traduction linguistique, selon laquelle «the aim of translation is to reinvent the source, so that, as in rhetorical theory, attention is focused on the active production of a new text endowed with its own affective powers and suited to the particular historical circumstances of its reception » (p. 30) s'applique également à la compilation, ainsi qu'à la traduction métaphorique de l'expérience vécue d'après le texte.
} 